Rev. Elev. Méd. vét. Pays trop., 1974, 27 (4) : 397-402

\title{
Enquête sérologique sur la péripneumonie et la brucellose bovines en Éthiopie
}

\author{
par J. DOMENECH (*) et P. C. LEFEVRE (*)
}

\begin{abstract}
RESUME
Une enquête sérologique effectuée en Ethiopie, province du Harrar, permet de déceler des taux d'infection de 1,78 p. 100 pour la péripneumonie bovine et de 0,43 p. 100 pour la brucellose bovine.

Par ailleurs, une fráquence élevée d'anticorps hétérophiles antihématies de mouton et de réactıons $\mathrm{HA}$ faussement positives est observée.
\end{abstract}

Après trois années consécutives de vaccination contre la péripneumonie et la peste bovine en Ethiopie, (PC 15 / JP 15), il a paru souhaitable de vérifier l'immunité du cheptel contre la peste bovine (15), et l'importance des porteurs de Mycoplasma mycoides, var. mycoides, agent de la péripneumonie bovine.

730 sérums ont été récoltés à cet effet, en 1973, dans la province du Harrar (Est éthiopien), district d'Alemaya.

Cette région fut choisie pour des raisons diverses :

— antécédents épidémiologiques notifiant l'existence de foyers de péripneumonie avant la mise en auvre de la prophylaxie;

- exemple de vaccination largement appliquée, dans une zone à élevage sédentaire exposée par le transit périodique d'animaux provenant de contrées infectées;

- relative facilité de collecte.

Mise à part la recherche des porteurs de germes, cette enquête a permis de constater

(*) Institut d'Elevage et de Médecine vétérinaire des Pays tropicaux, 10, rue P. Curie, 94700 MaisonsAlfort, France.

Mission vétérinaire française en Ethiopie.

Imperial Veterinary Institute, P.O. Box 19, DebreZeit, Ethiopie. l'importance, chez le zébu éthiopien, des anticorps hétérophiles anti-hématies de mouton, ainsi que de déceler un assez grand nombre de réactions HA faussement positives.

La recherche des bovins brucelliques fut ajoutée car aucune enquête n'avait été pratiquée dans la région.

\section{VACCIN UTILISE CONTRE LA PERIPNEUMONIE BOVINE}

C'est un vaccin lyophilisé préparé avec Mycoplasma mycoides, var. mycoides, souche $\mathrm{KH}_{3} \mathrm{~J}$ (96 $6^{\mathrm{e}}$ passage).

Le milieu de culture est le milieu F 66 de PROVOST (14), la digestion papaïnique de cœur de bœuf remplaçant la simple macération.

A la récolte, les titres en mycoplasmes viables, calculés par la méthode du Most Probable Number (M.P.N.) de TAYLOR (22), sont de 5,2 à $23 \cdot 10^{\circ}$ par $\mathrm{ml}$ de culture.

La lyophilisation se fait avec, pour seul support, du lait écrémé sec. $3,5 \mathrm{ml}$ de culture permettent d'obtenir 100 doses de vaccin lyophilisé, chaque dose titrant $5,2.10^{7}$ mycoplasmes viables. 
La perte en germes est due au processus de lyophilisation lui-même.

La vaccination est pratiquée, selon un rythme annuel, en association avec la vaccination antibovipestique (6).

Le lieu d'inoculation est le tissu sous-cutané en arrière de l'épaule.

Ce vaccin lyophilisé a été choisi par le gouvernement éthiopien car les conditions de transport sont souvent difficiles (7).

La souche $\mathrm{KH}_{3} \mathrm{~J}$ a été préférée à la souche $T_{1}$ en raison de sa parfaite innocuité, condition jugée indispensable pour assurer le succès le plus complet possible auprès d'éleveurs en général réticents.

\section{METHODES UTILISEES}

\section{Récolte des sérums}

730 échantillons de sang de zébus ont été recueillis par le Dr CAZALS en mars-avril 1973.

Les sérums sont numérotés et l'âge de I'animal, son sexe et le nombre de marques présumées dues à la vaccination relevés.

Les lieux de récolte, au nombre de sept, s'étalent dans tout le district d'Alemaya, sur des routes de passage d'animaux de commerce se dirigeant vers l'abattoir de Dire-Dawa.

Il est à noter que les éleveurs ont présenté leurs animaux à seule fin de prélèvement, sans qu'il $\mathrm{y}$ ait vaccination ou traitement concomitant (2).

Les résultats de l'enquête peuvent donc être considérés comme étant ceux que l'on obtient lors d'opérations de vaccination dans une région évoluée.

Ils ne sauraient être généralisés à toute l'Ethiopie.

\section{Méthodes sérologiques}

\section{a) Péripneumonie bovine}

- Les sérums ont été analysés par la méthode de fixation du complément de CAMPBELL et TURNER (11), aux dilutions $1 / 10,1 / 20$ et $1 / 40$.
Les résultats sont notés $0,+,++$, ,+++++++ , et les sérums positifs ++ au $1 / 10$ ou au-delà sont déclarés provenant d'animaux infectés.

- La réaction d'hémagglutination passive a été également étudiée car la comparaison HA avant absorption-HA après absorption, pratiquée sur quelques sérums a montré un fort pourcentage d'agglutinations dues à des anticorps hétérophiles anti-hématies de mouton, à des titres parfois très élevés.

Les hématies de mouton sensibilisées ont été aimablement fournies par le laboratoire de l'I.E.M.V.T., France (P. PERREAU).

Les dilutions initiales de sérum vont du $1 / 10$ au $1 / 320$.

On note la dernière dilution positive ++ .

\section{b) Brucellose bovine}

On a choisi la méthode de séro-agglutination lente en tubes $(1,19)$, avec des dilutions finales de sérum allant du 1/10 au 1/640.

L'antigène utilisé est préparé par l'Institut Merieux, France, à partir de Brucella abortus souche A 99: il agglutine $++1 / 650$ avec le sérum international.

Les sérums sont notés suspects aux dilutions $1 / 10$ et $1 / 40$ (30 à 60 U.I. $/ \mathrm{ml}$ ) et positifs au-delà $(100$ U.I./ml ou plus) $(1,19)$.

\section{RESULTATS}

\section{Péripneumonie bovine}

a) 730 sérums ont été analysés :

13 sérums sont positifs FC C-T, soit 1,78 p. 100.

Les résultats HA - FC C-T sont résumés dans le tableau I (p. 399).

La répartition des sérums positifs $\mathrm{FC}$, selon l'âge des animaux, est détaillée dans le tableau II (p. 399).

b) La comparaison HA avant absorption HA après absorption a été faite sur 507 sérums positifs HA, appartenant à un échantillonnage pris au hasard de 588 sérums.

Les résultats sont ceux du tableau III (p. 400). 
TABl.N`I. Péripneumonie:résultats des rếactions HA et FC C-T pratiquếes sur 730 sérums (Alemaya 1973).

\begin{tabular}{|c|c|c|c|c|c|c|}
\hline $\mathrm{HA}^{\mathrm{X}}$ & $\begin{array}{l}\text { Nombre } \\
\text { de sérums }\end{array}$ & $\begin{array}{c}\mathrm{EC} \\
\text { négatives }\end{array}$ & $\begin{array}{c}\text { FC } \\
\text { suspectes }\end{array}$ & $1 / 10$ & $\begin{array}{l}\text { ositives } \\
1 / 20\end{array}$ & $1 / 40$ \\
\hline 0 & 237 & 235 & 1 & 1 & 0 & 0 \\
\hline $1 / 10$ & 258 & 256 & 0 & 1 & 0 & 1 \\
\hline $1 / 20$ & 133 & 129 & 2 & 1 & 0 & 1 \\
\hline $1 / 40$ & 62 & 57 & 3 & 2 & 0 & 0 \\
\hline $1 / 80$ & 30 & 22 & 5 & 2 & 1 & 0 \\
\hline $1 / 160$ & 7 & 3 & 3 & 0 & 0 & 1 \\
\hline $1 / 320$ & 3 & 0 & 1 & 0 & 0 & 2 \\
\hline Total & 730 & 702 & 15 & & 13 & \\
\hline
\end{tabular}

* Titres donnés en dilution initiale de sérums, aprēs absorption sur hématies de mouton. H* + au $1 / 10$,

TABL. $\mathbb{N}^{\circ} \mathrm{II}$. Pêripneumonie: rêpartition des sérums positıfs FC C-T selon l'âge des animaux.

\begin{tabular}{|c|c|c|c|c|c|}
\hline Age & $\begin{array}{c}\text { Nombre } \\
\text { de sërums }\end{array}$ & $1 / 10$ & $\begin{array}{c}\text { FC positives } \\
1 / 20\end{array}$ & $\begin{array}{c}\text { Nombre de } \\
\text { positifs }\left(p \cdot 100^{\mathbf{x}}\right)\end{array}$ \\
\hline 1 à 3 ans & 214 & 1 & 0 & 1 & 0,93 \\
\hline 4 à 6 ans & 315 & 3 & 0 & 1 & 1,27 \\
\hline $\begin{array}{l}7 \text { ans et } \\
\text { plus }\end{array}$ & 201 & 3 & 1 & 3 & 3,50 \\
\hline Total & 730 & & 13 & & 1,78 \\
\hline
\end{tabular}

* Pourcentage calculé par rapport au nombre d'animaux dans la classe d'âge considérée.

\section{Brucellose bovine}

685 sérums ont été analysés :

- 15 sérums sont suspects, soit 1,9 p. 100.

- 3 sérums sont positifs, soit 0,43 p. 100 .

Les tableaux IV et $\mathrm{V}$ présentent les résultats selon l'âge et le sexe des animaux.

\section{DISCUSSION}

\section{Péripneumonie bovine}

\section{a) Importance des anticorps hétérophiles} anti-hématies de mouton

- Le tableau III montre à l'évidence le nombre très grand de sérums contenant des anticorps hétérophiles anti-hématies de mouton.

On observe, dans de très nombreux cas, des chutes de titre de 3,4 , voire 5 dilutions, entre l'HA avant et après absorption.

Ces résultats ne sont pas comparables avec ceux obtenus pour les zébus d'Afrique centrale et de l'Ouest (1 à 15 p. 100 des sérums contien- nent des anticorps anti-globules rouges de mouton, à des titres de $1 / 10$ à $1 / 40$ initial maximum) $(18,14)$.

On ne peut donc pratiquer la réaction HA, chez le zébu éthiopien, sans absorber au préalable les sérums par des hématies de mouton fraîches.

- Cette recherche systématique des anticorps hétérophiles anti-hématies de mouton a également permis de constater (tableau I) que, sur 102 sérums positifs HA, à des dilutions initiales de $1 / 40$ ou plus, 8 seulement sont confirmés par FC C-T.

En considérant que la FC est une des meilleures méthodes dans la recherche des porteurs de germes $(8,11,21)$, on voit qu'il y a un grand nombre de réactions $\mathrm{HA}$ faussement positives.

Les défaillances, par défaut, de la réaction HA sont, elles, normales et ont été maintes fois signalées $(11,14,18)$ : l'hémagglutination passive ne peut être utilisée dans le dépistage des infectés chroniques et latents en matière de péripneumonie bovine. 
TABL. ${ }^{\circ}$ III.Përipneumonie: comparaison des titres HA avant et après absorption par des hẻmaties de mouton.

\begin{tabular}{|c|c|c|c|}
\hline \multicolumn{2}{|c|}{ HA avant absorption ${ }^{*}$} & \multicolumn{2}{|c|}{ HA après absorption" } \\
\hline Titres & $\begin{array}{c}\text { Nombre } \\
\text { de sêrums }\end{array}$ & Titres & $\begin{array}{c}\text { Nombre } \\
\text { de sérums }\end{array}$ \\
\hline 0 & 81 & - & - \\
\hline $1 / 10$ & 67 & $\begin{array}{l}0 \\
1 / 10\end{array}$ & $\begin{array}{l}30 \\
37\end{array}$ \\
\hline $1 / 20$ & 90 & $\begin{array}{l}0 \\
1 / 10 \\
1 / 20\end{array}$ & $\begin{array}{l}24 \\
51 \\
15\end{array}$ \\
\hline $1 / 40$ & 117 & $\begin{array}{l}0 \\
1 / 10 \\
1 / 20 \\
1 / 40\end{array}$ & $\begin{array}{r}27 \\
61 \\
24 \\
5\end{array}$ \\
\hline $1 / 80$ & 148 & $\begin{array}{l}0 \\
1 / 10 \\
1 / 20 \\
1 / 40 \\
1 / 80\end{array}$ & $\begin{array}{r}18 \\
64 \\
46 \\
12 \\
8\end{array}$ \\
\hline $1 / 160$ & 49 & $\begin{array}{l}0 \\
1 / 10 \\
1 / 20 \\
1 / 40 \\
1 / 80 \\
1 / 160\end{array}$ & $\begin{array}{r}4 \\
0 \\
15 \\
22 \\
7 \\
1\end{array}$ \\
\hline Total & $\overline{588}$ & $\begin{array}{l}0 \\
1 / 10 \\
1 / 20 \\
1 / 40 \\
1 / 80 \\
1 / 160 \\
1 / 320\end{array}$ & $\begin{array}{r}5 \\
0 \\
3 \\
9 \\
12 \\
5 \\
2\end{array}$ \\
\hline
\end{tabular}

x titres donnés en dilution initiale du sêrum.

\section{b) Incidence de la péripneumonie}

Après trois années consécutives de vaccination massive du cheptel dans la région considérée, on peut, au vu des résultats, porter les mêmes conclusions que celles qui ont déjà été émises en Afrique ou en Australie :
- Les mesures de vaccination seules, comme moyen de lutte contre la péripneumonie bovine en région endémique, s'avèrent insuffisantes pour obtenir une éradication rapide $(7,10,20)$.

Dans le cas présent, il reste 1,78 p. 100 d'animaux infectés.

Signalons que l'emploi d'autres souches vaccinales $\left(\mathrm{T}_{1}\right.$ ou $\mathrm{V}_{5}$ par exemple) a, à cet égard, les mêmes effets $(9,20)$.

En conséquence, il est indispensable de poursuivre plus longtemps la vaccination, sous peine de voir réapparaître des foyers de péripneumonie.

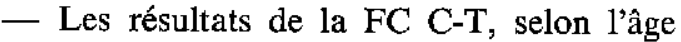
des animaux (tableau II), montrent que le pourcentage des réactions $\mathrm{FC}$ positives augmente avec l'âge.

Mais ceci peut être observé en toute occasion, même indépendamment de la vaccination : on ne peut donc affirmer que les jeunes zébus sont peu infectés parce qu'ils sont vaccinés depuis trois ans, et que les vieux sont très infectés $(3,50$ p. 100) parce que déjà atteints lorsque la campagne de prophylaxie médicale a commencé.

Aussi, nous n'apprécierons la valeur du vaccin $\mathrm{KH}_{3} \mathrm{~J}$ utilisé que par le fait que les cas cliniques de péripneumonie ont complètement disparu de la région, et ce malgré un transit certain d'animaux provenant de zones où sévit la maladie.

\section{Brucellose bovine}

Les résultats obtenus confirment que les caractères épidémiologiques de la brucellose bovine en Ethiopie sont les mêmes que partout

TABL. N`IV. Brucellose: répartition des sérums positifs et suspects selon l'âge des animaux.

\begin{tabular}{|c|c|c|c|c|c|}
\hline \multirow{2}{*}{$A g$ e } & \multirow{2}{*}{$\begin{array}{c}\text { Nombre } \\
\text { de } \\
\text { sérums }\end{array}$} & \multicolumn{2}{|c|}{ Suspects ${ }^{x}$} & \multicolumn{2}{|c|}{ Positifs } \\
\hline & & Nombre & Nombre $(p .100)$ & Nombre & Nombre $(\mathrm{p} \cdot 100)$ \\
\hline 1 a 3 ans & 201 & 3 & 1,5 & 0 & 0 \\
\hline 4 à 6 ans & 296 & 3 & 1,0 & 0 & 0 \\
\hline $\begin{array}{l}7 \text { ans et } \\
\text { plus }\end{array}$ & 188 & 7 & 3,7 & 3 & 1,6 \\
\hline Tota1 & 685 & 13 & 1,9 & 3 & 0,43 \\
\hline
\end{tabular}

к sérums +t $1 / 20$ et $1 / 40$. \%\# sérums $++1 / 80$ et $1 / 640$. 
TABL. N'V.Brucellose:répartitıon des sérums positifs et suspects selon le sexe des animaux.

\begin{tabular}{|c|c|c|c|c|c|}
\hline \multirow{2}{*}{ Sext } & \multicolumn{2}{c}{$\begin{array}{c}\text { Nombre } \\
\text { de } \\
\text { sérums }\end{array}$} & \multicolumn{2}{|c|}{ Suspects } & \multicolumn{2}{c|}{ positifs $^{\text {HH }}$} \\
\cline { 3 - 6 } & Nombre & Nombre (p.100) & Nombre & Nombre (p.100) \\
\hline Femelles & 385 & 12 & 1,7 & 3 & 0,78 \\
\hline Mâles & 300 & 1 & 0,3 & 0 & 0 \\
\hline Total & 685 & 13 & 1,9 & 3 & 0,43 \\
\hline
\end{tabular}

H sérums ++ $1 / 20$ et $1 / 40$, พअ sérums ++ 1/80 et au-dela.

ailleurs: différences de sensibilité du cheptel selon l'âge et le sexe (tableaux IV et $\mathrm{V}$ ).

On peut dire que la brucellose bovine, avec 0,43 p. 100 d'animaux infectés n'est pas un problème important dans cette partie de l'est éthiopien.

\section{CONCLUSION}

Cette enquête, portant sur 730 sérums prélevés dans l'est de l'Ethiopie, nous permet de constater que :

- la fréquence des sérums de zébus contenant des anticorps hétérophiles anti-hématies de mouton est très élevée;

- les réactions HA faussement positives sont, dans le diagnostic de la péripneumonie bovine, nombreuses;

- malgré une vaccination massive contre la péripneumonie pendant trois années con- sécutives, il reste 1,78 p. 100 d'animaux reconnus infectés par la méthode de fixation du complément.

Leur persistance rend nécessaire une prolongation de la lutte;

- la brucellose bovine, avec seulement $0,43 \mathrm{p}$. 100 d'animaux infectés, n'est pas un gros problème dans la région considérée.

\section{REMERCIEMENTS}

Nos plus vifs remerciements vont au $\mathrm{Dr}$ J. CAZALS sans qui cette étude n'aurait pu être faite, à M. M. VIGIER, codirecteur de l'Institut Veterinaire Imperial de Debré-Zeit, et à M. P. PERREAU, pour les conseils qu'ils nous ont prodigués.

Nous remercions également $M$. MESFIN WELDTSADIK pour son aide technique dans l'étude de la brucellose bovine.

\section{SUMMARY}

Serological survey on contagious bovine pleuropneumonia and bovine brucellosis in Ethiopia

A serological survey made in Ethiopia, in the Harrar province, reveals an infection rate of 1,78 p. 100 for contagious bovine pleuropneumonia and of 0,43 p. 100 for bovine brucellosis.

Besides, hererophilous antibodies against sheep red cells are often found, and falsely positive indirect haemagglutination test frequently occurs.

\section{RESUMEN}

Encuesta serologica sobre la perineumonia

y la brucelosis bovimas en Etiopia

Un estudio serológico en Etiopia, en la provincia de Harrar, demostró un porcentaje de infección de 1,78 p. 100 de los bovinos infectados con perineumonia contagiosa y de 0,43 p. 100 infectados con brucelosis.

Además, frecuentemente anticuerpos heterófilos contra los globulos rojos de ovino se encontraron y se observaron reacciones de hemaglutinación pasiva falsamente positivas. 


\section{BIBLIOGRAPHIE}

1. ALTON (G. G.) et JONES LOIS (M.). La brucellose : techniques de laboratoire. 1968. (Monographie O.M.S., $\mathrm{n}^{0}$ 55).

2. CAZALS (J.). Rapport de stage d'application: étude du district d'Alemaya, Ethiopie. MaisonsAlfort, France, I.E.M.V.T., 1973.

3. DAVIES (G.), MASIGA (W. N.), SHIFRINE (M.), READ (W.C.S.). The efficacy of the $T_{1}$ strain broth vaccine against contagious bovine pleuropneumonia: preliminary in contact trials Vet. Rec., 1968, 83 : 239-244.

4. DOUTRE (M.P.). Valeur de l'immunité conférée par les vaccins antipéripneumoniques lyophilisés. Rapport à la IVe réunion du groupe d'experts F.A.O./O.I.E./O.U.A. de la péripneumonie. Paris, 15-20 mars 1971.

5. DOUTRE (M.P.), CHAMBRON (J.) Valeur de l'immunité conférée par un vaccin antipéripneumonique lyophilisé préparé à l'aide de la souche $T_{1}$. Rev. Elev. Méd. vét. Pays trop., 1970, 23: 163-179.

6. DOUTRE (M.P.), CHAMBRON (J.), BOURDIN (P.). Valeur de l'immunité conférée par un vaccin mixte antibovipestique-antipéripneumonique lyophilisé préparé à l'aide de la souche $T_{1}$. Rev. Elev. Méd. vét. Pays trop., 1972, 25 (1): 1-14.

7. F.A.O./O.I.E./O.U.A. Rapport du sous-comité du groupe d'experts sur la péripneumonie, Lagos, 17-20 juillet 1970.

8. GOURLAY (R. N.). Comparison between some diagnostic tests for contagious bovine pleuropneumonia. J. comp. Path., 1965, 75: 97-109.

9. HUDDARD (J.E.). The use of $T_{1}$ broth culture in field control of contagious bovine pleuropneumonia. Confidential report. 30 juin 1966.

10. HUDDARD (J. E.) in HUDSON (J. R.). La péripneumonie contagieuse des bovidés. Rome, F.A.O., 1972. (Etudes agricoles de la F.A.O. n० 86), p. 75.

11. HUDSON (J. R.). La péripneumonie contagieuse des bovidés. Rome, F.A.O., 1972 (Etudes agricoles de la F.A.O. no 86).
12. HUDSON (J, R.). Contagious bovine pleuropneumonia : the immunizing value of the attenuated strain $\mathrm{KH}_{3} \mathrm{~J}$. Aust. vet. J., 1965, 41 : $43-49$.

13. HUDSON (J.R.). Contagious bovine pleuropneumonia: development of a satisfactory and safe vaccine. Rapport à la III session du groupe d'experts F.A.O./O.I.E./O.U.A. de la péripneumonie bovine. Khartoum, Soudan, 12-13/02/ 1967.

14. Institut d'Elevage et de Médecine vétérinaire des Pays tropicaux, laboratoire de Farcha, Tchad. Rapport d'activité, année 1966, 2.

15. LEFEVRE (P. C.), DOMENECH (J.). Contrôle sérologique de l'immunité conférée par la vaccination antibovipestique en Ethiopie. Rev. Elev. Méd. vét. Pays trop., 1974, 27 (2) : 177-181.

16. LINDLEY (E. P.). An immunity test in cattle to compare two C.B.P.P. vaccines. Rapport à la IIIe session du groupe d'experts F.A.O./O.I.E./ O.U.A. de la péripneumonie bovine. Khartoum, Soudan, 12-15 février 1967.

17. ORUE (J.). Valeur de l'immunité conférée par les vaccins antipéripneumoniques lyophilisés. Rapport à la IVe session du groupe d'experts F.A.O./O.I.E./O.U.A. de la péripneumonie bovine. Paris, 15-20 mars 1971.

18. PERREAU (P.) ,PROVOST (A.), REGNOULT $\left(R_{1}\right)$, ORUE $(\mathrm{J})$. Valeur de la réaction d'hémagglutination indirecte dans la péripneumonie bovine. Rev. Elev. Méd. vét. Pays trop., 1964, 17 (1): 1-14.

19. RENOUX (G.), GAUMONT (R.). Méthodes de diagnostic biologique des brucelloses animales. Ann. Nutr. Alim., 1966, 20 (1) : 1-51.

20. PROVOST (A.). Etat actuel de l'immunisation active dans la péripneumonie bovine. Rapport au XVII Congrès Mondial Vétérinaire. Paris, 1967.

21. SHIFRINE (M.), GOURLAY (R. N.). Evaluation of diagnosis tests for contagious bovine pleuropneumonia. Bull. epizoot. Dis. Afr., 1967, 15 : 7-10.

22. TAYLOR. Theory and practice in experimental bacteriology, 1965, p. 204. 